\title{
CORRECTION
}

\section{Correction to: Trends in insulin-like growth factor-1 levels after bariatric surgery: a systematic review and meta-analysis}

Mohammad Hassan Sohouli, Mansoureh Baniasadi, Raheleh Nabavizadeh, Elma Izze da Silva Magalhães, Heitor O. Santos, Somaye Fatahi and Mojtaba Lotfi iD

(c) The Author(s), under exclusive licence to Springer Nature Limited 2022

International Journal of Obesity (2022) 46:1079; https://doi.org/10.1038/s41366-022-01083-6

Correction to: Int J Obes https://doi.org/10.1038/s41366-02101051-6, published online 17 January 2022
The original version of this article contained a mistake in an author name. The correct full name is "Elma lzze da Silva Magalhães" instead of "Elma Izze da Silva Magalhãesd". The original article has been corrected. 